Flores-Rodríguez, J.; Ramírez-Macías, G. (2021) Individual Defense in Handball: Influence of Task Constraints. Revista Internacional de Medicina y Ciencias de la Actividad Física y el Deporte vol. 21 (82) pp. 269-284 Http://cdeporte.rediris.es/revista/revista82/artconstrenimiento1244.htm DOI: https://doi.org/10.15366/rimcafd2021.82.005

\title{
ORIGINAL
}

\section{DEFENSA INDIVIDUAL EN BALONMANO: INFLUENCIA DE LOS CONSTREÑIMIENTOS DE LA TAREA}

\section{INDIVIDUAL DEFENSE IN HANDBALL: INFLUENCE OF TASK CONSTRAINTS}

\author{
Flores-Rodríguez, J. ${ }^{1}$ y Ramírez-Macías, G. $^{2}$ \\ ${ }^{1}$ Graduado en Ciencias de la Actividad Física y Deporte. Universidad de Sevilla (España). \\ josefloresrodríguez@live.es \\ ${ }^{2}$ Profesor titular, Departamento Educación Física y Deporte. Universidad de Sevilla (España). \\ grm@us.es
}

Código UNESCO / UNESCO code: 5899 Otras especialidades pedagógicas (Pedagogía no lineal) / Other pedagogical specialties (Non-linear pedagogy) Clasificación Consejo de Europa / Council of Europe classification: 5. Didáctica y metodología / Didactics and methodology

Recibido 7 de mayo de 2019 Received May 7, 2019 Aceptado 20 de mayo de 2019 Accepted May 20, 2019

\section{RESUMEN}

El objetivo del presente trabajo fue analizar la influencia que ejercen determinados constreñimientos o limitaciones de la tarea en el aprendizaje de habilidades defensivas en balonmano. Empleando un diseño observacional y mediante el uso de un instrumento de observación ad hoc, se registraron los comportamientos realizados por 14 jugadores $(M=14,6)$ a lo largo de seis sesiones, mientras disputaron 18 partidos en situación de juego reducido, empleando ambos equipos un sistema defensivo individual. Los partidos se disputaron en presencia de tres constreñimientos, y para estudiar su influencia en los comportamientos de los jugadores, se utilizó la técnica de coordenadas polares. Se encontró que la prohibición del bote facilita la defensa de desmarques, mientras que la obligatoriedad de recibir y pasar en movimiento facilita la defensa de fintas, interceptaciones, así como, la realización de doblajes y deslizamientos. Estos resultados pueden ayudar a los entrenadores a optimizar sus programas de entrenamiento.

PALABRAS CLAVE: balonmano, pedagogía no lineal, constreñimiento, juegos reducidos 


\section{ABSTRACT}

The aim of this study was to analyze the influence of certain task constraints, on the learning of defensive handball skills. By applying the observational methodology and through the use of an ad hoc observation instrument, the behaviors performed by 14 players $(M=14,6)$ were registered over six sessions, while they played 18 games in a small-sided game situation using both teams an individual system defense. Matches were played under the influence of three task constraints and to study their influence on the behavior of the players, it was used the polar coordinate technique. It was found that the prohibition of the bound facilitates the defense of clearing out, whereas the obligation of getting and passing the ball in movement enables the defense of faints, as well as, the realization of steals, helps and follows to another player. These results can help coaches optimize their training programs.

KEY WORDS: handball, non-linear pedagogy, constraint, small-sided games

\section{INTRODUCCIÓN}

En los deportes de equipo, como el balonmano, las acciones deportivas se desarrollan en entornos de gran incertidumbre (múltiples interacciones entre compañeros y adversarios, bajo la influencia de numerosos factores que se condicionan mutuamente), siendo poco probable que los jugadores se enfrentan dos veces a la misma situación (Seirul·lo, 2017; Varela, Ureña, Ruiz \& Alarcón, 2010). La naturaleza incierta e imprevisible de las acciones, junto con el poco tiempo disponible para su realización, enfatizan, por un lado, la importancia de que el jugador desarrolle su capacidad de adaptación y de actuación en entornos tremendamente cambiantes y, por otro, cuestionan la existencia de programas motores y representaciones mentales previamente almacenas en el cerebro, tal y como se asume en los modelos de enseñanza tradicionales (Balagué, Torrents, Pol, \& Seirul·lo, 2014). En los últimos años diversos estudios, realizados en el ámbito de la neurociencia, parecen confirmar este planteamiento, al demostrar como determinadas acciones motrices ni se planifican (Soon, Brass, Heinze, \& Haynes, 2008), ni tampoco se inhiben mediante decisiones elaboradas conscientemente (Filevich, Künh, \& Haggard, 2013). Estos hallazgos, sugieren que el proceso de entrenamiento de las acciones propias de los deportes colectivos sea predominantemente implícito y no consciente, en lugar de explícito y consciente tal y como se concibe en las metodologías tradicionales (Balagué et al., 2014). En las metodologías tradicionales predominan tareas de entrenamiento excesivamente analíticas y estructuradas (Robles-Rodríguez, Giménez \& Abad, 2011), que poco se parecen a los contextos cambiantes propios del partido; este hecho ha sido, y sigue siendo, objeto de debate pues surgen dudas respecto a la transferencia de los aprendizajes adquiridos en este tipo de tareas (Chow \& Atencio, 2014; Nathan, Salimin, \& Shahril, 2017; Seirul·lo, 2017).

Así, en los últimos años están emergiendo con fuerza propuestas metodológicas, como la pedagogía no lineal, que difieren sustancialmente de los métodos de 
enseñanza tradicionales. La pedagogía no lineal ofrece a los investigadores un potente marco teórico para el estudio los deportes de equipo (Chow, Davids, Hristovski, Araújo, \& Passos, 2011); a la vez que, para entrenadores y profesores de educación física, se consolida como una firme_alternativa a los métodos tradicionales, ofreciendo mejores resultados en el entrenamiento de las habilidades propias de los deportes de equipo (Clark, Mcewan, \& Christie, 2018). Esta pedagogía, fundamentada en postulados de la teoría general de sistemas y de la psicología ecológica, concibe el proceso de enseñanza-aprendizaje en términos de relaciones funcionales y adaptativas entre un sistema (deportista) y su entorno (tarea de aprendizaje o situación de juego real), en lugar de pretender que el deportista reproduzca perfectamente un determinado modelo de ejecución técnica (Araújo \& Davids, 2011; Renshaw \& Chow, 2018).

En concreto, considera que el aprendizaje emerge como consecuencia de la exploración activa de la tarea por parte del jugador, pues a medida que actúa percibe directamente sin necesidad de representaciones mentales, sus afforadances o posibilidades de actuación que una determinada situación presenta para él (Passos, Araújo, Davids, \& Shuttleworth, 2008). El jugador y equipo, mientras participa en la tarea, modifica y/o crea patrones de conducta que le ayudan a resolver con éxito los constreñimientos o limitaciones presentes (Araújo, Davids, \& Hristovski, 2006; Chow \& Atencio, 2014).

Al respecto, es importante subrayar que los constreñimientos tienen un papel esencial en la pedagogía no lineal, pues al reducir el número de comportamientos disponibles para el deportista, favorecen la realización de determinadas habilidades técnicas y tácticas, en detrimento de otras (Balagué, Pol, Torrents, Ric, \& Hristovski, 2019). En concreto, se distinguen tres grandes tipos de constreñimientos: los del ambiente (condiciones climatológicas, presencia de espectadores, etc.), los propios del jugador o equipo que participa (nivel de habilidad, características antropométricas y funcionales, etc.) y los de la tarea (objetivos, reglas, materiales utilizados, espacio de juego, etc.) (Correia, Carvalho, Araújo, Pereira, \& Davids, 2018).

La actuación sobre los constreñimientos de la tarea (selección, modificación y/o eliminación) es considerada como la principal herramienta del entrenador para provocar la emergencia de determinados comportamientos que faciliten la consecución de los objetivos de aprendizaje propuestos (Cantos y Moreno, 2019). Con el propósito de afinar el proceso de manipulación y selección de los constreñimientos a introducir en las tareas, el entrenador se enfrenta a un doble desafío, por un lado, debe conocer la influencia que ejercen los constreñimientos en el comportamiento de sus jugadores y, por otro, debe estimar las posibles acciones que realizarán para solucionar con éxito la tarea (Renshaw \& Chow, 2018). Por ello, es importante identificar tanto las tendencias generales de comportamiento (patrones de conducta que son habituales en determinadas situaciones), como las limitaciones y/o necesidades específicas que presentan sus jugadores a nivel individual, grupal y colectivo.

Con el objetivo de conocer la influencia ejercida por determinados constreñimientos de la tarea, en los últimos años están proliferando estudios centrados en deportes de equipo como: rugby (Cantos \& Moreno, 2019), fútbol 
(Ric et al., 2017), baloncesto (Esteves et al., 2015) o voleibol (Paulo, Davids, \& Araújo, 2018), que han aportado una valiosa información para el trabajo de investigadores y entrenadores. Sin embargo, sigue siendo necesario profundizar sobre este aspecto clave: conocer la influencia de los constreñimientos de la tarea en los comportamientos realizados por los deportistas durante el entrenamiento, de forma que se pueda responder a las siguientes preguntas ¿cuáles son los constreñimientos de la tarea más relevantes para facilitar la adquisición de determinadas habilidades? ¿cómo sabemos que la manipulación de ciertos constreñimientos de la tarea facilita o dificulta a los jugadores la realización de determinados comportamientos? ¿afecta por igual a todos los jugadores la presencia de un determinado constreñimiento? (Correia et al., 2018).

A las preguntas anteriores se suma el hecho de que no existe conocimiento empírico sobre la implantación de los postulados de la pedagogía no lineal en el aprendizaje de las habilidades individuales y colectivas del balonmano. Por todo ello, el presente trabajo tiene como objetivo conocer la influencia que ejercen determinados constreñimientos, aplicados en partidos de balonmano disputados en una situación de juego modificada, en la realización de habilidades defensivas individuales (asignación de marcaje, interceptación, marcaje en línea de tiro, marcaje a jugador con balón que intenta fintar, desposesión en bote y blocaje) y colectivas (deslizamiento, cambio de oponente y doblaje), valorando si esta influencia se expresa de igual manera en todos los jugadores. La obtención de evidencias al respecto, podría ayudar a la optimización de los programas de entrenamiento, favoreciendo que los entrenadores puedan seleccionar los constreñimientos de la tarea más relevantes para la consecución de los objetivos de aprendizaje propuestos y evitando que esta selección esté basada exclusivamente en la intuición o en el conocimiento subjetivo (Renshaw \& Chow, 2018).

\section{MATERIAL Y MÉTODOS}

La metodología observacional es especialmente apropiada para el estudio de los comportamientos de los jugadores en la situación real de juego (Aragón, Lapresa, Arana, Anguera, \& Garzón, 2016). De acuerdo con los objetivos de investigación propuestos, se optó por un diseño observacional Seguimiento//diográfico/Multidimensional (S//M) (Anguera, Blanco-Villaseñor, Hernández-Mendo, \& Losada, 2011). Seguimiento, debido a que se realizó un análisis inter-sesional e intra-sesional que aportó información sobre la frecuencia de las acciones realizadas; Idiográfico, al estudiar los comportamientos de varios jugadores que al formar parte de un mismo equipo (sistema) funcionaron como unidad; y Multidimensional porque se estudian diversos niveles de comportamientos defensivos tanto individuales como colectivos.

\subsection{PARTICIPANTES}

El proyecto de investigación se formuló de acuerdo con el código de conducta y los principios éticos establecidos en la Declaración de Helsinki y en la declaración de Derechos Humanos, obteniendo la aprobación por parte del comité ético de la Universidad de Sevilla. Participaron 14 jugadores que forman 
parte de un equipo de balonmano cadete masculino, $(M=14,6$; $D T=0,4)$ que compite en una liga provincial de Sevilla (España). Los jugadores, con más de cuatro años de experiencia previa en la práctica de balonmano ( $M=4,2$ años; $D T=$ $0,8)$, dedicaban a la práctica del balonmano cuatro horas semanales, distribuidas en cuatro sesiones, más un partido correspondiente a la competición oficial.

Los 14 jugadores fueron distribuidos en dos equipos, de nivel equilibrado según el criterio del entrenador, que no se modificaron a lo largo de las seis sesiones (dos sesiones a la semana) en las que se desarrolló la investigación. Se disputaron 18 partidos (tres partidos por sesión) en una situación de juego modificada. Cada partido tuvo una duración de 10 minutos y se disputó en un terreno de juego de 24 metros de largo por 14 metros de ancho. Los equipos compitieron con 5 jugadores a la vez y utilizaron obligatoriamente un sistema defensivo individual.

\subsection{INSTRUMENTO DE OBSERVACIÓN}

Las unidades de observación fueron todas las secuencias defensivas de ambos equipos, es decir, cuando el equipo analizado no tiene la posesión del balón. Para registrar los comportamientos defensivos (individuales y colectivos) más relevantes para la consecución de los objetivos de investigación propuestos, se construyó un instrumento de observación ad hoc (Tabla 1). El instrumento de observación combinó el formato de campo con un sistema de categorías exhaustivas y mutuamente excluyentes. Esta combinación permite aprovechar los puntos fuertes de ambos instrumentos, por un lado, el sistema de categorías ofrece consistencia teórica, mientras que el formato de campo aporta flexibilidad a la hora de recoger la diversidad de comportamientos realizados por los jugadores, permitiendo una adaptación perfecta a las características de los participantes (Anguera \& Hernández-Mendo, 2013).

Tabla 1 - Instrumento de observación empleado en el estudio.

\begin{tabular}{|c|c|}
\hline Criterio & Categorías \\
\hline $\begin{array}{l}\text { Constreñimiento } \\
(\mathrm{CON})\end{array}$ & $\begin{array}{l}\text { Constreñimientos presentes cuando se desarrolla la acción defensiva: } \\
\text { - La acción analizada se desarrolla sin la presencia de } \\
\text { constreñimientos (LIB). } \\
\text { - La acción analizada se desarrolla con la prohibición del bote (NBT). } \\
\text { - La acción analizada se desarrolla con la obligatoriedad de pasar y } \\
\text { recibir en carrera (MVT). }\end{array}$ \\
\hline Equipo (EQU) & $\begin{array}{l}\text { La acción defensiva analizada corresponde al equipo: } \\
\text { - Naranja (NAR). } \\
\text { - Azul (AZU). }\end{array}$ \\
\hline $\begin{array}{l}\text { Habilidades } \\
\text { individuales } \\
\text { en defensa } \\
\text { (HIN) }\end{array}$ & $\begin{array}{l}\text { Acción individual que realiza el defensor responsable del atacante que } \\
\text { finaliza la posesión de balón: } \\
\text { - El atacante realiza un lanzamiento después de recibir sólo, debido } \\
\text { a un error en la asignación del marcaje de los atacantes sin balón } \\
\text { (dos defensores marcan al mismo atacante o un defensor no marca } \\
\text { a nadie) (ERR). } \\
\text { - El defensor, por medio de la interceptación, recupera la posesión de } \\
\text { balón tras impedir que se complete un pase (ITR). } \\
\text { El defensor, realizando un marcaje en línea de tiro, se opone a un } \\
\text { desmarque del atacante con la intención de recibir el balón en buena } \\
\text { situación para marcar gol (EPL). }\end{array}$ \\
\hline
\end{tabular}


- $\quad$ El defensor marca a un jugador con balón que realiza una acción de finta (UX1).

- El defensor intenta recuperar la posesión de balón ante un atacante que bota (RBT).

- $\quad$ El defensor realiza una acción de blocaje ante un lanzamiento lejano (BLC).

- $\quad$ El defensor del jugador que realiza el lanzamiento, ha acudido a la ayuda de un compañero que ha sido superado (AYD).

Colaboración defensiva, entre al menos dos defensores, durante la posesión de balón.

- Doblaje a un compañero que ha sido superado (DBL).

Habilidades - Cambio de oponente: ante atacantes que colaboran por medio de colectivas cruce, permuta o bloqueo (CMB).

en defensa $(\mathrm{HCL})$

- Deslizamiento: ante atacantes que atacantes que realizan colaboran por medio de cruce, permuta o bloqueo (DLZ).

- Otra colaboración distinta a las anteriores (OTR).

- No se produce ningún tipo de colaboración entre los defensores $(\mathrm{SIN})$.

Modo en el que finaliza el ataque:

- El ataque finaliza con un lanzamiento (LZM).

Finalización $\quad$ - El ataque finaliza con una recuperación de balón por parte de la (FIN) defensa (RCP).

- $\quad$ El ataque finaliza con la señalización de un golpe franco o con una interrupción del juego, sin haya cambio en la posesión del balón (GFC).

Se inicia la fase de defensa analizada después de:

Inicio del ataque (INI)
- $\quad$ Saque de puerta (PRT).

- $\quad$ Saque de golpe franco o saque de banda (SQE).

- Pérdida de balón (CTQ).

\subsection{INSTRUMENTOS DE REGISTRO Y ANÁLISIS}

La grabación de los 18 partidos fue realizada utilizando una cámara Sony FDRAX33 ${ }^{\circ}$, situada a 4 metros de altura detrás de una de las porterías, garantizando un óptimo plano de visión. Una vez grabados los partidos, se aplicó el instrumento de observación por medio del programa informático DARTFISH $5.5 \otimes$, que actuó como instrumento de registro y codificación de todas las defensas. Una vez codificados los comportamientos, se procedió al análisis de la calidad de dato mediante el cálculo del coeficiente Kappa, realizado mediante el paquete informático SDIS-GSEQ 4.0. (Bakeman \& Quera, 1995). Posteriormente, por medio del programa HOISAN 1.2 (Hernández-Mendo, López-López, Castellano, Morales-Sánchez, \& Pastrana, 2012), se aplicó la técnica de coordenadas polares que permitió conocer los patrones de conducta expresados por los jugadores, para representarlos gráficamente utilizando la aplicación Snowflake $0.2 ®$. Por último, señalar que, como requisito previo a la aplicación de la técnica de coordenadas polares, fue necesaria la realización del análisis secuencial utilizando para ello el programa SDIS-GSEQ 4.0.

\subsection{PROCEDIMIENTO}

Los 14 jugadores fueron distribuidos en dos equipos, de nivel equilibrado según el criterio del entrenador, que no se modificaron a lo largo de las seis sesiones (dos sesiones a la semana) en las que se desarrolló la investigación. Se 
disputaron 18 partidos (tres partidos por sesión), de 10 minutos de duración en una situación modificada de juego ( 5 vs. 5, en un terreno de juego de 24 metros de largo por 14 metros de ancho, con un área rectangular situada a 6 metros de la portería) donde ambos equipos utilizaron obligatoriamente un sistema defensivo individual. Las situaciones de juego modificadas cumplen con los postulados de la pedagogía no lineal en el diseño de tareas, ya que, al conservar la estructura y la esencia del deporte real, permiten que los jugadores puedan desarrollar las habilidades individuales y colectivas en contextos similares a los de la competición (Chow, Davids, Button, \& Renshaw, 2015).

Los 18 partidos se jugaron bajo la influencia de los tres constreñimientos de la tarea (seis partidos por cada constreñimiento analizado), cuya influencia en los comportamientos defensivos fue objeto de análisis en este estudio. Los primeros seis partidos se disputaron sin la presencia de ningún constreñimiento adicional (LIB), los siguientes seis partidos se disputaron en presencia del constreñimiento prohibición del bote (NBT) y, por último, los seis partidos finales se disputaron bajo la influencia del constreñimiento de la tarea: pasar y recibir en carrera (MVT). En ningún momento, ni antes, ni durante, ni después de los partidos, el entrenador proporcionó a los jugadores ningún tipo de información o indicaciones relacionadas con el desarrollo del juego para evitar influir en los comportamientos realizados.

La observación y codificación de las acciones fue realizada por dos observadores, ambos con titulación de entrenador nacional de balonmano, entrenados previamente en la utilización de los instrumentos de observación y de registro. El control de calidad del dato del registro a nivel interobservador se llevó a cabo mediante el coeficiente de concordancia Kappa de Cohen, obteniéndose $k=0,90$. La fiabilidad intraobservadores, considerando todos los criterios de manera conjunta, fue superior a 0,93 para ambos observadores. Los resultados obtenidos que pueden ser considerados almost perfect según la clasificación de Landis \& Koch (1977).

\section{RESULTADOS}

\subsection{ANÁLISIS DE COORDENADAS POLARES}

El análisis de coordenadas polares es una valiosa herramienta que permite analizar los patrones de conducta que emergen durante la práctica del balonmano (Flores-Rodríguez \& Anguera, 2018). Como paso previo a su realización, se requiere el cálculo de los residuos ajustados mediante la aplicación del análisis secuencial. El objetivo del análisis secuencial es identificar las relaciones significativas, tanto a nivel prospectivo como retrospectivo, entre los distintos comportamientos que son analizados, pues tanto la perspectiva prospectiva como la retrospectiva, deben ser tenidas en cuenta en la representación gráfica (Gorospe \& Anguera, 2000). Una de las categorías analizadas asume el rol de conducta focal, al ser considerada como generadora, "hacia adelante" (perspectiva prospectiva) y "hacia atrás" (perspectiva retrospectiva), de las asociaciones con las otras conductas estudiadas, que adquieren el rol de conductas condicionadas (Tarragó et al., 2017). Por medio 
del cálculo del estadístico $Z_{\text {sum }}=\frac{\sum Z}{\sqrt{n}}$, una potente técnica de reducción de datos, (Sackett, 1980) se conoce tanto la fuerza de la asociación, indicada por la longitud del vector, como la naturaleza de la misma, que puede ser de activación o inhibición, señalada por el cuadrante en el que queda ubicada. Cuando la asociación es representada en el cuadrante I, indica una relación de activación mutua entre la conducta focal y la condicionada, sin embargo, cuando se representa en el cuadrante III, se expresa una relación de mutua inhibición. La representación gráfica en el cuadrante II señala que la conducta focal inhibe a la condicionada, mientras es activada por ella; situación inversa a la obtenida cuando la asociación se ubica en el cuadrante IV, pues en este caso la conducta focal activa a la condicionada, a la vez que es inhibida por ella (Anguera et al., 2011).

En los siguientes apartados se comentan las asociaciones significativas, aquellas con una longitud $>1,96(p<0,05)$ representadas en los distintos mapas de coordenadas polares ubicados en las tabla 2. La tabla consta de nueve mapas, distribuidos en tres filas y tres columnas, donde actúan como conducta focal los distintos constreñimientos presentes en el desarrollo de los partidos; en la primera fila se analizan los comportamientos expresados cuando los partidos se desarrollan sin la presencia de constreñimientos (LIB); en la segunda fila cuando el partido se juega con la prohibición del bote (NBT) y en la tercera fila se analizan los comportamientos cuando es obligatorio pasar y recibir el balón en carrera (MVT). Como conductas condicionadas, actuarán todas las categorías pertenecientes a los criterios: habilidades individuales (HIN) y habilidades colectivas en defensa (HCD). Por último, referente a las columnas, la situada a la izquierda analiza los comportamientos de ambos equipos de manera conjunta; la central aborda los comportamientos realizados por el equipo naranja (NAR), y en la situada a derecha quedan representadas las asociaciones significativas del equipo azul (AZU). El orden de mención de las distintas asociaciones representadas será de mayor a menor intensidad.

\subsection{PARTIDOS SIN CONSTREÑIMIENTOS ADICIONALES (LIB)}

En los partidos disputados sin la presencia de ningún constreñimiento (LIB), el análisis conjunto de los comportamientos de ambos equipos señala una relación de mutua activación (Cuadrante I) con AYD (defensor del jugador que realiza el lanzamiento, ha acudido a la ayuda de un compañero que ha sido superado), ERR (error en la asignación del marcaje), RBT (el defensor 
Tabla 2 - Mapas de coordenadas polares obtenidos actuando como conductas focales los constreñimientos introducidos en las tareas.

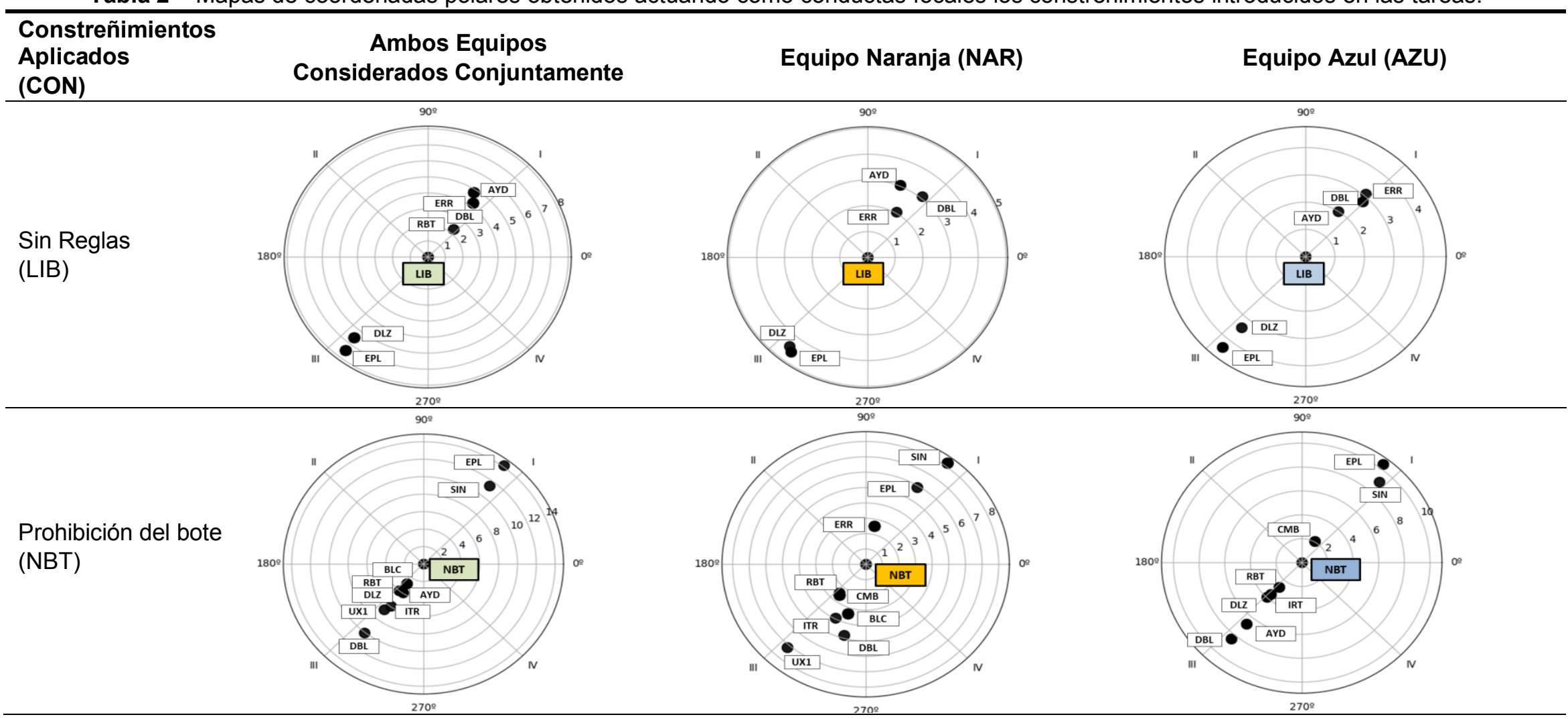


Rev.int.med.cienc.act.fís.deporte - vol. 21 - número 82 - ISSN: 1577-0354

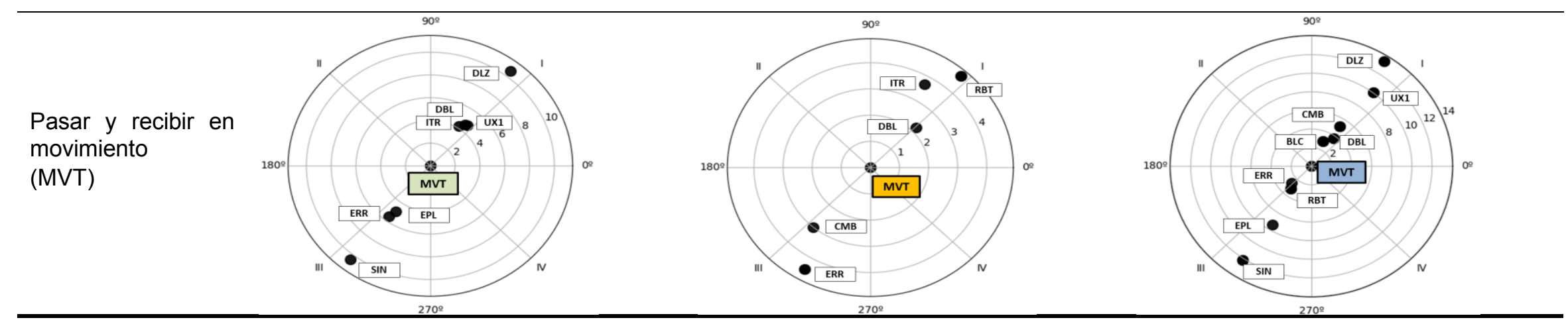


intenta recuperar la posesión de balón ante un atacante que bota) y DBL (doblaje a un compañero que ha sido superado). A la misma vez, se observa asociación de mutua inhibición (Cuadrante III) con las conductas condicionadas EPL (el defensor se opone a un desmarque del atacante con la intención de recibir el balón en buena situación para marcar gol) y DLZ (deslizamiento).

Cuando se analizan los comportamientos de ambos equipos por separado, vemos que el equipo naranja (NAR) presenta una asociación de mutua activación con AYD, DBL y ERR y una relación de inhibición mutua con EPL y DLZ. Por su parte, el equipo azul (AZU) muestra una activación reciproca con las conductas condicionadas: ERR, DBL AYD, e Inhibición mutua con EPL y DLZ.

\subsection{CONSTREÑIMEINTO: PROHIBICIÓN DEL BOTE (NBT)}

En las acciones defensivas desarrolladas bajo la presencia del constreñimiento NBT, al analizar los comportamientos de ambos equipos de manera conjunta, podemos observar una relación de activación recíproca con EPL y SIN (no se produce ningún tipo de colaboración entre los defensores) y una asociación de inhibición mutua con: DBL, UX1 (el defensor se opone a la realización de una finta por parte del atacante que finaliza), ITR (el defensor recupera la posesión de balón tras impedir que se complete un pase), DLZ, AYD, RBT y BLC (el defensor realiza una acción de blocaje ante un lanzamiento lejano).

En el análisis de ambos equipos por separado, el equipo naranja presenta una asociación de activación mutua con las conductas SIN, EPL y ERR y una relación de inhibición mutua con las categorías: UX1, DBL ITR, BLC y CMB (cambio de oponente) y RBT. El equipo azul por su parte, tiene una relación de activación mutua con EPL, SIN y CMB y una asociación de inhibición mutua con DBL, AYD, DLZ, ITR y RBT.

\subsection{CONSTREÑIMIENTO: PASAR Y RECIBIR EN MOVIMIENTO (MVT)}

La influencia del constreñimiento MVT, cuando se analizan los comportamientos de ambos equipos de manera conjunta, se expresa en una asociación de mutua activación con las habilidades defensivas: DLZ, DBL, UX1 e ITR, a la misma vez que emerge una relación de inhibición mutua con: SIN, ERR y EPL.

En el análisis de los comportamientos de ambos equipos por separada, la presencia del constreñimiento MVT, provoca en el equipo naranja una activación mutua con RBT, ITR y DBL y una inhibición mutua con ERR y CMB. Sin embargo, para el equipo azul, el mismo constreñimiento provoca una activación mutua con DLZ, UX1, CMB, DBL y BLC, a la misma vez que presenta una asociación de mutua inhibición con SIN, EPL, RBT y ERR.

\section{DISCUSIÓN}

El objetivo de esta investigación fue conocer la influencia que ejercen determinados constreñimientos, introducidos en el desarrollo de partidos de balonmano disputados en situación de juego modificada, en la realización de 
habilidades defensivas individuales (asignación de marcaje, interceptación, marcaje en línea de tiro, marcaje a jugador con balón que intenta fintar, desposesión en bote y blocaje) y colectivas (deslizamiento, cambio de oponente y doblaje), valorando si esta influencia se expresa de igual manera en todos los jugadores. Por medio de la técnica de coordenadas polares se analizaron, por un lado, las conductas de todos los jugadores, para conocer las tendencias generales $\mathrm{y}$, por otro, las de cada equipo, para conocer sus tendencias específicas.

Debido a la ausencia de estudios similares con los que discutir los resultados, confrontaremos los diferentes patrones de conducta encontrados en presencia de los constreñimientos investigados. En primer lugar, hay que subrayar que, debido al carácter reactivo de la mayoría de las habilidades defensivas analizadas, los comportamientos defensivos están condicionados por las acciones realizadas por el equipo atacante. Por este motivo, cuando los partidos se desarrollan sin la presencia de ningún constreñimiento, los atacantes utilizan principalmente acciones con balón para superar a la defensa, en detrimento de la realización de desmarques o cambios de posiciones, situación que provoca que las habilidades defensivas RBT, AYD y DBL se activen, a la vez que se inhiben EPL y DLZ. Asimismo, el desarrollo del juego sin constreñimientos, parece favorecer la aparición de ataques rápidos dificultando la distribución de marcajes ERR por parte de los defensores.

Por su parte, la prohibición del bote (presencia del constreñimiento NBT) limita los desplazamientos con balón a los atacantes, situación que provoca la emergencia de otros comportamientos (sin balón) para marcar gol como los desmarques, lo que provoca una asociación de activación mutua muy fuerte con la habilidad defensiva individual EPL. Asimismo, la influencia en el desarrollo del juego que provoca este constreñimiento, hace que no aparezcan habilidades colectivas defensivas SIN.

En los partidos disputados bajo la influencia del constreñimiento MVT, debido a la obligatoriedad que tienen los atacantes de pasar y recibir en movimiento, se favorecen las acciones de finta a la vez que fomentan una mayor movilidad e intercambio de posiciones por parte de los atacantes. Este estilo de juego, provoca que se activen las habilidades UX1 y DBL, para contrarrestar las fintas, a la misma vez que se active la habilidad colectiva DLZ, como respuesta al intercambio de posiciones de los jugadores atacantes. Asimismo, el constreñimiento MVT, al limitar el tiempo disponible para realizar el pase, podría explicar que la aparición en defensa de comportamientos orientados a la interceptación de pases (ITR).

Respecto a la habilidad colectiva cambio de oponente (CMB), no se ha encontrado asociación de mutua activación con ninguno de los constreñimientos empleados en esta investigación, este hecho podría deberse a que el sistema defensivo individual, empleado por ambos equipos en todos los partidos, ofrece un contexto poco favorable para su utilización.

Teniendo en cuenta lo anterior, podemos advertir que las habilidades defensivas realizadas, individuales y colectivas, son fruto de la interacción entre los 
constreñimientos individuales (características individuales de los jugadores y equipos) y los constreñimientos introducidos en las tareas. Esto explica la emergencia de patrones de conducta específicos, según el grupo de jugadores que sean analizados (Balagué et al., 2019). De esta forma, se pone de manifiesto la relación de interdependencia existente entre todos los elementos presentes en el momento en el que se realiza la acción deportiva, pues cambios en uno de estos elementos acarrean cambios en el comportamiento final del sistema (es decir, del jugador) (Flores-Rodríguez \& Anguera, 2018). Por todo ello, los resultados obtenidos evidencian que la manipulación de los constreñimientos de la tarea puede ser una potente herramienta para el entrenador a la hora de provocar y/o inhibir la aparición de determinados comportamientos individuales y colectivos (Cantos \& Moreno, 2019; Renshaw \& Chow, 2018).

\section{CONCLUSIONES}

El presente estudio contribuye, en parte, a cubrir la necesidad de obtener conocimiento empírico sobre la influencia (facilitación o inhibición) ejercida por determinados constreñimientos de la tarea, en los comportamientos realizados por los jugadores.

Para los participantes en este estudio, se ha demostrado que hay unos constreñimientos más relevantes que otros para la adquisición de determinados comportamientos. La utilización del constreñimiento MVT facilita la realización de las habilidades defensivas DLZ, UX1, DBL, ITR, a la misma vez que dificulta la aparición de SIN, ERR y EPL. Por su parte, la presencia del constreñimiento NBT ha facilitado la aparición de comportamientos EPL, SIN y ha bloqueado la aparición de los comportamientos DBJ, UX1, DLZ, ITR, RBT, BLC. Asimismo, el sistema defensivo individual no favorece la realización de cambios de oponente $\mathrm{CMB}$, pues ninguno de los constreñimientos empleados ha activado esta habilidad colectiva.

Aunque existen tendencias generales de comportamiento, también aparecen comportamientos específicos en los grupos estudiados. Esto señala que la influencia ejercida por los constreñimientos no es igual para todos los jugadores, subrayándose la necesidad de cuidar aspectos como la composición de grupos (a nivel intra e intergrupal) en los entrenamientos, o la manipulación o selección de constreñimientos específicos para cada grupo.

Para mejorar la potencialidad de los resultados obtenidos en esta investigación, sería recomendable comprobar el influjo que ejercen los constreñimientos estudiados en otros participantes (jugadores de diferente edad, sexo y nivel de pericia), así como en otro tipo tareas o partidos (diferente número de jugadores por equipo, diferentes dimensiones del terreno de juego, utilización de otros sistemas defensivos, etc.). De igual manera, habida cuenta del potencial explicativo del análisis de coordenadas polares, sería recomendable en investigaciones futuras, estudiar la influencia que ejercen otros constreñimientos en los comportamientos realizados en las distintas fases del juego. 


\section{REFERENCIAS BIBLIOGRÁFICAS}

Anguera, M. T., Blanco-Villaseñor, A., Hernández-Mendo, A., \& Losada, J. L. (2011). Diseños observacionales: Ajuste y aplicación en psicología del deporte. Cuadernos de Psicología del deporte, 11(2), 63-76.

Anguera, M. T., \& Hernández-Mendo, A. (2013). La metodología observacional en el ámbito del deporte. E-Balonmano.com: Revista de Ciencias del Deporte, 9(3), 135-160.

Aragón, S., Lapresa, D., Arana, J., Anguera, M. T., \& Garzón, B. (2016). Tactical behaviour of winning athletes in major championship 1500-m and 5000-m track finals. European Journal of Sport Science, 16(3), 279-286. https://doi.org/10.1080/17461391.2015.1009494

Araújo, D., \& Davids, K. (2011). What Exactly is Acquired during skill acquisition? $\begin{array}{llll}\text { Journal of } & \text { Consiousness }\end{array}$ https://doi.org/10.3171/JNS/2008/108/5/0950

Araújo, D., Davids, K., \& Hristovski, R. (2006). The ecological dynamics of decision making in sport. Psychology of Sport and Exercise, 7(6), 653-676. https://doi.org/10.1016/j.psychsport.2006.07.002

Balagué, N., Pol, R., Torrents, C., Ric, A., \& Hristovski, R. (2019). On the Relatedness and Nestedness of Constraints. Sports Medicine, 5(6), 1-10. https://doi.org/https://doi.org/10.1186/s40798-019-0178-z

Balagué, N., Torrents, C., Pol, R., \& Seirul·lo, F. (2014). Entrenamiento integrado. Principios dinámicos y aplicaciones. Apunts Educación Física y Deportes, 116(2), 60-68. https://doi.org/DOI: http://dx.doi.org/10.5672/apunts.20140983.es.(2014/2).116.06

Cantos, J., \& Moreno, F. J. (2019). Pedagogía no lineal como método de enseñanza de los comportamientos tácticos en los deportes de equipo, aplicación al rugby. Retos, 35(1), 402-406.

Chow, J. Y., \& Atencio, M. (2014). Complex and nonlinear pedagogy and the implications for physical education. Sport, Education and Society, 19(8), 1034-1054. https://doi.org/10.1080/13573322.2012.728528

Chow, J. Y., Davids, K., Button, C., \& Renshaw, I. (2015). Nonlinear Pedagogy in Skill Acquisition: An Introduction. Abingdon: Routledge.

Chow, J. Y., Davids, K., Hristovski, R., Araújo, D., \& Passos, P. (2011). Nonlinear pedagogy: Learning design for self-organizing neurobiological systems. New $\begin{array}{llll}\text { Ideas in } & \text { 29(2), }\end{array}$ https://doi.org/10.1016/j.newideapsych.2010.10.001

Clark, M. E., Mcewan, K., \& Christie, C. J. (2018). The effectiveness of constraints-led training on skill development in interceptive sports: A systematic review. International Journal of Sports Science \& Coaching, 14(2), 241-254. https://doi.org/10.1177/1747954118812461

Correia, V., Carvalho, J., Araújo, D., Pereira, E., \& Davids, K. (2018). Principles of nonlinear pedagogy in sport practice. Physical Education and Sport Pedagogy, 24(2), 117-132. https://doi.org/10.1080/17408989.2018.1552673

Esteves, P. T., Silva, P., Vilar, L., Travassos, B., Duarte, R., Arede, J., \& Sampaio, J. (2015). Space occupation near the basket shapes collective behaviours in youth basketball. Jounal of Sports Sciences, 34, 1557-1563. https://doi.org/10.1080/02640414.2015.1122825

Filevich, E., Künh, S., \& Haggard, P. (2013). There Is No Free Won ' t: 
Antecedent Brain Activity Predicts Decisions to Inhibit. PLoS ONE, 8(2), e53053. https://doi.org/. doi:10.1371/ journal.pone.0053053

Flores-Rodríguez, J., \& Anguera, M. T. (2018). Patrón de juego en balonmano según el jugador que ocupa la posición de central. Apunts Educación Física y Deportes, 134(4), 110-123. https://doi.org/DOI: http://dx.doi.org/10.5672/apunts.2014-0983.es.(2018/4).134.08

Gorospe, G., \& Anguera, M. T. (2000). Modificación de la técnica clásica de coordenadas polares mediante un desarrollo distinto de la retrospectividad: Aplicación al tenis. Psicothema, 12(SUPPL. 2), 279-282.

Hernández-Mendo, A., López-López, J. A., Castellano, J., Morales-Sánchez, V., \& Pastrana, J. L. (2012). HOISAN 1 . 2 : Programa informático para uso en Metodología Observacional. Cuadernos de Psicología del Deporte, 12(1), 55-77. https://doi.org/https://doi.org/10.4321/S1578-84232012000100006

Landis, J. R., \& Koch, G. G. (1977). The Measurement of Observer Agreement for Categorical Data. Biometrics, 33(1), 159-174.

Nathan, S., Salimin, N., \& Shahril, M. I. (2017). A comparative analysis of badminton game instructions effect of non-linear pedagogy and linear pedagogy. J. Fundam. Appl. Sci., 9(6S), 1258-1285. https://doi.org/10.4314/jfas.v9i6s.94

Passos, P., Araújo, D., Davids, K., \& Shuttleworth, R. (2008). Manipulating Constraints to Train Decision Making in Rugby Union. International Journal of Sports Science and Coaching, 3(1), 125-140. https://doi.org/10.1260/174795408784089432

Paulo, A., Davids, K., \& Araújo, D. (2018). Co-adaptation of ball reception to the serve constrains outcomes in elite competitive volleyball. International Journal of Sports Science and Coaching, 13(2), 253-261. https://doi.org/10.1177/1747954117722727

Renshaw, I., \& Chow, J. Y. (2018). A constraint-led approach to sport and physical education pedagogy. Physical Education and Sport Pedagogy, 24(2), 103-116. https://doi.org/10.1080/17408989.2018.1552676

Ric, A., Torrents, C., Gonçalves, B., Torres-Ronda, L., Sampaio, J., \& Hristovski, R. (2017). Dynamics of tactical behaviour in association football when manipulating players' space of interaction. PLoS ONE, 12(7), e0180773. https://doi.org/10.1371/journal.pone.0180773

Robles-Rodríguez, J., Giménez Fuentes-Guerra, F.J., \& Abad Robles, M.T. (2011). Metodología utilizada en la enseñanza de los contenidos deportivos durante la E.S.O. Revista Internacional de Medicina y Ciencias de la Actividad Física y del Deporte, 10(41), 35-57.

Sackett, G. P. (1980). Lag Sequential Analysis as a data reduction technique in social interaction research. En D. B. Sawin, R. C. Hawkins, L. O. Walker, \& J. H. Penticuff (Eds.), Exceptional infant. Psychosocial risks in infantenvironment transactions (pp. 300-340). New York: Brunner/Mazel.

Seirul-lo, F. (2017). ¿Entrenamiento estructurado en los deportes de equipo? En El entenamiento en los deportes de equipo (pp. 16-40). Barcelona: Mastercede.

Soon, C. S., Brass, M., Heinze, H., \& Haynes, J.-D. (2008). Unconscious determinants of free decisions in the human brain. Nature Neuroscience, 11(5), 543-545. https://doi.org/10.1038/nn.2112

Tarragó, R., Iglesias, X., Lapresa, D., Anguera, M. T., Ruiz-Sanchis, L., \& Arana, 
J. (2017). Análisis de las relaciones diacrónicas en los comportamientos de éxito y fracaso de campeones del mundo de esgrima utilizando tres técnicas complementarias. Anales de Psicología, 33(3), 471-485. https://doi.org/http://dx.doi.org/10.6018/analesps.33.3.271041

Valera, S., Ureña, N., Ruiz \& Alarcón, F. (2010). La enseñanza de los deportes colectivos en Educación Física en la E.S.O. Revista Internacional de Medicina y Ciencias de la Actividad Física y el Deporte 10(40), 502-520.

Número de citas totales / Total references: 30 (100\%)

Número de citas propias de la revista / Journal's own references: 2 (6,67\%)

Rev.int.med.cienc.act.fís.deporte - vol. 21 - número 82 - ISSN: 1577-0354 